\title{
Reisen mit „Handicap“ - Was ist zu beachten?
}

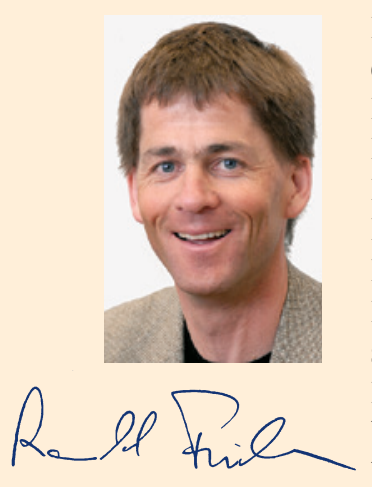

Liebe Kolleginnen und Kollegen,

die Überschrift dieses Editorials ist nicht ganz zu ernst zu nehmen, zumindest nicht in Bezug auf den einen der beiden Beiträge in diesem Heft, auf die sie sich bezieht. Stefan Hagmann beleuchtet in seinem Artikel die Problematik von Reisen mit Kindern in Malariagebiete. Wenn die Probleme von Reisen mit Kindern und mögliche Empfehlungen in einer Runde von Ärzten angesprochen werden, gibt es ganz unterschiedliche Reaktionen. Eine Gruppe lehnt jegliches, mögliches Risiko für Kinder durch eine Reise rundweg ab, sie favorisieren für Kinder (oft bis hin zu Jugendlichen) die heimische Umgebung, allenfalls noch Reisen ins benachbarte Ausland werden akzeptiert. Eine weitere Gruppe akzeptiert durchaus Reisen auch in weiter entfernt gelegene Gebiete, aber nur mit ausreichender Infrastruktur und guter medizinischer Versorgung, besonders bei kleinen Kindern. Und oft nur ein kleiner Teil kann sich mit dem Gedanken anfreunden, dass Kinder auch in Gegenden mitgenommen werden, in denen vielleicht keine optimale medizinische Versorgung möglich ist oder in denen sie besonderen Risiken, wie zum Beispiel bei einem Höhenaufenthalt oder eben in Malariagebieten, ausgesetzt sind. Dabei wird jedoch oft vergessen, dass es auch in diesen Gebieten Kinder gibt, die - wie im Beitrag von Hagmann betont - aus diesen Ländern zu uns kommen und dann zu Besuch wieder zurückfahren. Aber auch beruflich bedingte Reisen erfordern manchmal die Begleitung durch Kinder. Letztlich muss jeder - nach ausführlicher Beratung - für sich selbst und die ihm anvertrauten Kinder entscheiden, welches Risiko er akzeptiert. Daher ist es so wichtig, dass wie im vorliegenden Beitrag detailliert Risiken und Nebenwirkungen einer Malariaexposition für Kinder dargestellt werden.

Diese detaillierte Information ist auch wichtig für Patienten, bei denen eine Antikoagulation notwendig ist und die trotzdem verreisen möchten. Jürgen Ringwald stellt in seinem Beitrag die aktuellen Möglichkeiten der Antikoagulation vor und beleuchtet detailliert die Vor- und Nachteile der Vitamin-K-Antagonisten, der direkt wirkenden Antikoagulanzien sowie der niedermolekularen Heparine. Ob im Einzelfall eine Umstellung der Präparate notwendig ist, ist eben nicht nur von der Pharmakologie abhängig, sondern auch von Reiseziel, Reisedauer oder weiteren Begleiterkrankungen.

Dass nicht nur Reisen Risiken aufweisen, zeigt sich im Beitrag von Marija Stojkovic über die zystische und alveoläre Echinokokkose. Gerade die alveoläre Echinokokkose (oft auch Fuchsbandwurm genannt) breitet sich in Europa weiter aus und ist leider nur schwer zu kontrollieren, da zunehmend Füchse oder Kojoten auch in städtische Umgebungen vordringen.

Kinder laufen daher auch bei uns Gefahr, mit den Eiern von Echinococcus multilocularis in Kontakt zu kommen und sich zu infizieren.

Mit diesen kurzen Hinweisen auf das vorliegende Heft habe ich Sie hoffentlich neugierig gemacht und bin sicher, dass Sie auch mit dieser Ausgabe der FTR zufrieden sind.

Mit herzlichen Grüßen

PD Dr. Rainald Fischer, München 